Original article

\title{
TOXOPLASMA GONDII INFECTIONS IN PIGS FROM SOUTH BENIN AND ASSESSMENT OF BREEDERS' KNOWLEDGE ABOUT TOXOPLASMOSIS
}

\author{
A. B. N. TONOUHEWA ${ }^{1}$, Y. AKPO ${ }^{1,3}$, P. SESSOU ${ }^{1}$, C. SALANON ${ }^{1}$, \\ G. L. APLOGAN ${ }^{4}$, M. N. ASSOGBA ${ }^{2}$, I. A. K. YOUSSAO ${ }^{2} \&$ S. FAROUGOU ${ }^{1}$
}

${ }^{1}$ Communicable Disease Research Unit (URMaT), University of Abomey-Calavi, Cotonou, Benin; ${ }^{2}$ Department of Animal Heath and Production, University of Abomey-Calavi, Cotonou, Benin; ${ }^{3}$ Laboratory of Ecology, Health and animal Production, Faculty of Agronomy, University of Parakou, Parakou, Benin; ${ }^{4}$ Laboratory for Veterinary Diagnosis and Serosurveillance of Animal Diseases, Ministry of Agriculture, Livestock and Fisheries, Benin

\section{Summary}

Tonouhewa, A. B. N., Y. Akpo, P. Sessou, C. Salanon, G. L. Aplogan, M. N. Assogba, I. A. K. Youssao \& S. Farougou, 2020. Toxoplasma gondii infections in pigs from south Benin and assessment of breeders' knowledge about toxoplasmosis. Bulg. J. Vet. Med., 23, No 2, $248-256$.

Toxoplasma gondii is a ubiquitous apicomplexan parasite that causes toxoplasmosis, a cosmopolitan zoonosis responsible for abortion and congenital malformation in animals and humans. Pigs can be a reservoir for $T$. gondii and act as one of the sources for parasite transmission to humans. Currently, there are no data about T. gondii infection in any animal in Benin. After the report of several cases of abortions and reproductive problems in pigs in several livestock farms in the southern region of Benin, this study was carried out to investigate the occurrence of this zoonotic parasite in pigs. A total of 184 pig serum samples have been collected in two municipalities and sent to the laboratory for the detection of anti-T. gondii IgG antibodies by ELISA indirect method. The results showed the presence of anti-Toxoplasma gondii $\mathrm{IgG}$ in $23 \%\left(\mathrm{IC}_{95}=22.56-23.44\right)$ of pigs $(42 / 184)$. Age, sex, and race did not seem to affect the frequency of this infection. Moreover, reproductive failure has been reported on many farms. These were cases of stillbirths in $40 \%$ of farms followed by neonatal mortality (36\%) and abortion cases which were frequently observed in $27 \%$ of farms. $T$. gondii is present in pigs from south Benin and could be responsible for reported abortions in the areas. In addition, the raw and undercook meat of pigs could be important source of Toxoplasma gondii infection in humans from south Benin.

Key words: abortion, Benin, ELISA, IgG, pig, Toxoplasma

\section{INTRODUCTION}

The protozoan parasite Toxoplasma gondii can infect all mammals and birds on all continents (Lindsay \& Weiss, 2008; Dubey, 2016). It is an intracellular coccidian 
of apicomplexan phylum, the etiologic agent of toxoplasmosis. This disease is a major zoonosis responsible for abortion and congenital malformation in humans and animals. Among animal species, sheep, goats and pigs are the most affected. In pigs, the infection usually occurs postnatally by ingestion of oocysts from contaminated environment and ingestion of infected tissues of animals, or prenatally by transplacental transmission of the parasite (Dubey, 2009). The disease can then be severe or fatal with abortion cases and mortality especially in piglets. In humans, who can get infected by the consumption of food contaminated with oocysts excreted by cats or by undercook meat containing tissue cysts, most infections are asymptomatic, except in immunocompromised people and pregnant women (Tenter et al., 2000). In Africa, most studies on animal toxoplasmosis have shown the variability in the level of infection for different animal species and areas, with high prevalence in chickens and low prevalence in cattle (Tonouhewa et al., 2017). In pigs, a prevalence varying from $9 \%$ to $39 \%$ was reported (Tonouhewa et al., 2017).

Benin is an African country where approximately half of the human population is infected, with an estimated prevalence of $48 \%$ for toxoplasmosis in the southern region (Ogouyèmi-Hounto et al., 2014). Studies carried out between 1997 and 2014 showed a decreasing proportion of $63 \%$ and $48 \%$ of seropositive pregnant women (Akpovi et al., 1998; Ogouyèmi-Hounto et al., 2014). That implies a gradual increase in the proportion of women not immunised against toxoplasmosis among Beninese population, therefore exposed to congenital toxoplasmosis. Otherwise in the country, pig farming is one of the main activities in the breeding sector and pork is highly appreciated by consumers particularly in the southern region. Unfortunately, the pork industry is confronted with many health problems, including the failure of reproductions on farms and hygienic and sanitary quality of pork destined for human consumption, now that pigs are considered as an important intermediate host of many parasites including $T$. gondii. Human toxoplasmosis acquired after pork consumption has been reported in some countries (Dubey \& Jones, 2008; Wang et al., 2012; Hill \& Dubey, 2016). To date, there seems to be no report on the presence of $T$. gondii among any animal species in Benin, although the infection has been reported in humans.

The present study was therefore undertaken to provide current information on the presence of $T$. gondii infection in pigs raised for human consumption in south Benin, and is the first to examine $T$. gondii prevalence in food animals in the study area.

\section{MATERIALS AND METHODS}

\section{Ethical standards}

This study has been approved by the Committee for Animal Experiments of the Department of Animal Production and Health of Polytechnic School of University of Abomey-Calavi of Benin. The methods used in this protocol involving animals were in accordance with the ethical standards of this institution.

\section{Study area}

This study was conducted in the Atlantic and Littoral departments of southern Benin between September 2016 and March 2017. 
Toxoplasma gondii infections in pigs from south Benin and assessment of breeders' knowledge ....

\section{Survey and sampling}

Following the reports on the frequency of abortions in pig farms in southern Benin, a cross-sectional survey was conducted in 100 livestock farms in the Atlantic Department located in the region. The objective was to assess current farming practices, the magnitude of reproduction failure and knowledge of breeders about toxoplasmosis, a zoonosis responsible for abortion in pigs. In farms chosen for the survey with the collaboration of the veterinary service of the Ministry of Agriculture, Breeding and Fishery (MAEP/ Benin), the information about breeding practice, history of reproduction, knowledge on toxoplasmosis and the role of cats as vectors of zoonosis transmission to animals and humans has been collected from breeders.

Subsequently, a seroepidemiological survey on $T$. gondii infection in pigs was conducted between October 2016 and January 2017 in two municipalities to estimate the level of infection in pig in the area. For sampling, a minimum sample size of 97 was determined in order to estimate the prevalence with at least $10 \%$ precision at $50 \%$ expected prevalence and $95 \%$ level of confidence according to Thrusfield (2005). A total of 184 pig samples were collected using a simple random sampling technique. The sampling was made at livestock farms identified in the municipalities of Abomey-Calavi and the slaughterhouse of Cotonou where animals raised in the region are slaughtered for human consumption.

\section{Serological examination}

Pig sera was tested using commercial indirect ELISA Kits (ID Screen Toxoplasmosis Indirect Multi-Species from ID Vet Innovative Diagnostic, Montpellier, France) to determine the presence of
Toxoplasma specific IgG antibodies, resulting from infection with $T$. gondii according to the manufacturer's instruction. This kit has been used for detecting specific antibodies to $T$. gondii in pigs, sheep, and other mammals including cats and dogs. For serological examination, a 96well plate was prepared containing the test and control specimens, before transferring them into the ELISA microplate using a multichannel pipette, in order to avoid differences in incubation times between specimens. According to the manufacturers' recommendation, sera with sample-topositive $(\mathrm{S} / \mathrm{P} \%)$ ratio less than or equal to $40 \%$ were considered negative, between 40 and $50 \%$ were considered doubtful and greater than or equal to $50 \%$ - positive. The test was validated if the mean value of the positive control optical density (ODPC) was greater than 0.350 $($ ODPC $>0.350)$ and the ratio of the mean O.D. values of the positive and negative controls (ODPC and ODNC) was greater than 3.5 (ODPC/ODNC > 3.5). Doubtful samples were repeated for certainty.

\section{Statistical analysis}

The results were expressed in percentages. Data were statistically analysed using chisquare test or exact Fischer test to compare seroprevalence values between modalities of different variables. Data analyses were performed by Epi-Info 7 for Windows (Epi-Info 7, CDC, USA). The differences were considered statistically significant at $\mathrm{P}<0.05$.

\section{RESULTS}

\section{Pig farms management}

The surveyed breeders consisted of $96 \%$ men and 4\% women. The enrolment rate was $27 \%$ with $13.04 \%$ at the primary le- 
vels, $65.21 \%$ and $14.81 \%$ at the secondary and university levels respectively. Most of the farms had a pig population between 5 and 216 with an average of $55.2 \pm 49.70$. Out of all farms, $62 \%$ practiced confined animal production system compared to $38 \%$ with outdoor system. The different reared breeds consisted of $\mathrm{Me}$ tis $(61 \%)$ followed by local breed $(20 \%)$ and Large White, Landrace (19\%). With regard to animal feeding, complete food rations were offered in $40 \%$ of farms, agro-food by-products associated with food waste - in $50 \%$, while $10 \%$ of breeders served leftovers to their animals. The drinking water was $75 \%$ well-water or uncovered tank, and $90 \%$ of breeders used self-medication in case of pig diseases, while $10 \%$ waited for the advice of a veterinarian to administer health care.

\section{Reproductive disorders}

Table 1 shows reproduction disorders observed at the surveyed farms. The most frequent reproductive failure was stillbirth $(40 \%)$ and neonatal mortality observed in $36 \%$ of farms. Otherwise, abortions cases were frequently observed in $27 \%$ of farms. Toxoplasmosis is recognised as an abortive disease, so these reproductive disorders could be related to this zoonotic infection.

\section{Knowledge about toxoplasmosis}

Regarding the knowledge of pig breeders about toxoplasmosis (Table 1), most breeders had no information about this parasitic zoonosis. Only $6 \%$ of pig farmers have heard of toxoplasmosis at least once and knew that infection could be responsible for abortion in pregnant women. All respondents $(100 \%)$ were unaware that pigs can contract toxoplasmosis. Only $5 \%$ of breeders knew that the cat is the definitive host of this parasite and can transmit $T$. gondii infection to pigs or humans. The presence of resident or wandering cats has been observed in more than half of the farms and respondents were not aware of the role played by the cat in the epidemiology of this parasitic zoonosis. Finally, on $29 \%$ of the farms measures of rodent control were not applied, although rats were recognised as T. gondii reservoir.

Table 1. Knowledge of breeders about toxoplasmosis

\begin{tabular}{llll}
\hline Questions & Response & Number & P \\
\hline Reproduction disorders on farms in the & Neonatal mortality & 36 & $\leq 0.05$ \\
& Stillbirths & 40 & \\
last 6 months & Abortions & 27 & $\leq 0.01$ \\
Have you ever read, heard or seen any & No & 94 & $\leq 0.01$ \\
information about Toxoplasmosis? & Yes & 6 & $\geq 0.05$ \\
Can pigs contract toxoplasmosis? & No & 100 & $\leq 0.05$ \\
& Yes & - & \\
Do cats run on the farm? & No & 58 & $\leq 0.01$ \\
& Yes & 42 & \\
Can cat transmit toxoplasmosis to pigs & No & 94 & \\
or humans? & Yes & 5 & \\
Rodent control at the farm & No & 29 & 71 \\
\end{tabular}


Toxoplasma gondii infections in pigs from south Benin and assessment of breeders' knowledge ....

Table 2. Seropositivity of Toxoplasma gondii infection in pigs from south Benin

\begin{tabular}{lrcc}
\hline Variable & Number of samples & Number of positive samples & Prevalence \\
\hline Sex & & & \\
Female & 106 & 26 & 24.52 \\
Male & 78 & 16 & 20.51 \\
\hline Age, years & & 7 & \\
$<1$ & 33 & 35 & 21.22 \\
$>2$ & 151 & & 23.17 \\
\hline Breed & & 11 & 22.00 \\
Local & 50 & 31 & 23.00 \\
Exotic & 133 & & \\
\hline Municipality & & 25 & 26.00 \\
Ab-Calavi & 96 & 17 & 23.00 \\
Cotonou & 88 & 42 & \\
\hline Total & 184 & & \\
\hline
\end{tabular}

\section{T. gondii infection among pigs}

In the present study, antibodies against $T$. gondii were detected in $23 \%$ of pigs (42/186). This implies a continuous exposure of this animal species to the oocyst of T. gondii excreted in the environment by cats in study area where stray cat presence was reported. The highest seroprevalence was observed in sows: $25 \%(26 / 106)$ compared to boars $(21 \% ; 16 / 78) . T$. gondii infection was higher in AbomeyCalavi municipality $(26 \% ; 25 / 95)$ than in Cotonou municipality $(19.31 \% ; 18 / 88)$. In addition, higher levels of infection were recorded in animals older than 1 year $(23.11 \%)$ compared to younger ones $(21.22 \%)$. Moreover, there was no significant difference between the prevalence levels observed according to the different variables at the $5 \%$ level of statistical significance. This result shows that the spatial distribution of the infection may be related to the circulation of stray cats, which are abundant in southern Benin.

\section{DISCUSSION}

This is the first epidemiological report of T. gondii infection in any animal in Benin. The results show that this zoonotic parasite was present among pigs in Benin and can be transmitted to humans, which confirms the high seroprevalence of human toxoplasmosis recorded in south Benin, attributed to the fact that food animals can be reservoirs for $T$. gondii and act as one of the sources for parasite transmission to humans.

There are different methods to evaluate pig infection by $T$. gondii. Among these diagnostic methods, serological techniques are the most commonly used (Gamble et al., 2005). For the present study, the ELISA test was used. This serologic technique is not specific to all animal species, but is used among many animal species including pigs for seroepidemiological studies because of the specificity and high sensitivity (Gamble et al., 2005; Dubey, 2009). Villena et al. (2012) and Villard et al. (2016) showed that there was a high agreement between the ELISA 
technique, the modified agglutination test and the reference test, e.g. the bioassay in mice in detection of Toxoplasma gondii infections in animals.

The results of this study revealed a relatively high level of pig infection by $T$. gondii in southern Benin. Indeed, about one-fifth of pigs were infected by $T$. gondii, which highlights the presence and circulation of this parasite in the farms investigated. This result confirms the frequency of reproduction failures recorded on farms in the areas. Toxoplasmosis is generally asymptomatic in farm animals. However, in pigs, the infection is responsible for reproductive disorders such as neonatal mortality, stillbirths and abortions (Dubey, 2009). Unfortunately, most breeders (96\%) had no knowledge about this disease and its zoonotic potential, while animal husbandry practices carried out during the survey are likely to favour this infection. Many factors can affect pig infection by $T$. gondii. Among these factors, the practice of outdoor breeding favours animals contact with $T$. gondii oocysts contaminating the environment, as environmental conditions are favourable for the survival of $T$. gondii oocysts in the soil. The presence of cats and the lack of rodent control in pig farms promotes the spread of the parasite (Kijlstra et al., 2008; Dubey, 2009). However, during the survey, most of these factors were recorded on pig farms. Benin has a humid subtropical climate with an average annual temperature of $27.5{ }^{\circ} \mathrm{C}$ and a high humidity from March to November (Aw climate type) according to the KöppenGeiger classification (Peel et al., 2007). These environmental conditions are favourable for the survival of $T$. gondii oocysts throughout the year (Dubey, 2016).

Other studies have been carried out on the seroprevalence of toxoplasmosis in pigs from West African countries. Ayinmode \& Olaosebikan (2013) have studied the prevalence of $T$. gondii infections in pigs from Ibadan, Nigeria. The results showed a level of infection (25\% among 100 pigs) comparable to that obtained in Benin for the present study $(23 \%, 43 / 184)$. This can be explained by the geographical position and the similar climatic conditions prevailing in these two countries. In fact, Benin and Nigeria are border countries and there is a humid subtropical climate in the southern region of the two countries where the studies were carried out, and pig management system is comparable in the different West African countries. In Bobo-Dioulasso (Burkina Faso) Bamba et al. (2016) obtained a prevalence of $29 \%$ among 300 pigs, higher than that recorded in the present study. This disparity could be related to different populations of pigs sampled. Indeed, the authors studied the seroprevalence of $T$. gondii infections only in wandering pigs more exposed to the risk of contamination by $T$. gondii oocysts than animals raised in confinement system. However, for the present study, exotic pigs reared in confinement generally were taken into account, as well as wandering pig raised in extensive system. In Ghana and Ethiopia, however, infection levels (39\% among 641 pigs and $32 \%$ among 402 pigs respectively) were significantly higher compared to those obtained for the present study (ArkoMensah et al., 2000; Gebremedhin et al., 2015). Moreover, Hove et al. (2005) reported very low seroprevalence $(9 \%$ among 97 pigs) of $T$. gondii infections in the same species in Zimbabwe, while in Madagascar similar infection levels to those obtained in this study $(23 \%$ among 250 pigs) were recorded by Rakotoharinome et al. (2012), which shows a high 
Toxoplasma gondii infections in pigs from south Benin and assessment of breeders' knowledge ....

variability in the seroprevalence of infections by country and region.

Although toxoplasmosis is a zoonosis, responsible for abortions in women and reproductive disorders in pig farming, it is still a little-known disease for most breeders in Benin. In this study, only $6 \%$ of pig breeders had heard of it but most of them were unaware that animals could contract the infection, resulting in reproductive disorders in infected sows. In addition, given the magnitude of the reproductive history observed in some farms and the level of $T$. gondii infection recorded in this study, it is likely that this history was due in part to this zoonosis. Finally, toxoplasmosis is not the only disease that can cause reproductive disorders in pigs, other additional studies are needed to confirm this hypothesis and show the link between this disease and reproductive problems observed.

Human toxoplasmosis has been described in newborn, pregnant and immunocompromised individuals in south Benin (Zannou et al., 2004; Avode et al., 2005). This situation can therefore be related to the presence and circulation of $T$. gondii in domestic animals including pig, that maintain the parasite's life cycle in the environment and thus promote the transmission of infection to humans (Tenter et al., 2000; Dubey, 2016). Therefore, pork consumption can result in the acquisition of toxoplasmosis if the pork is raw or undercooked. This is the first time that T.gondii infection is described in animals from Benin. So, further work is required to assess infection level among other animal species and the risk factors for human and animal infections.

\section{CONCLUSION}

The parasite $T$. gondii is present in domestic animals from Benin, especially pigs. This is the first epidemiological report of $T$. gondii prevalence in any animals from Benin. It is shows that $T$. gondii is present in food animals in this country and that their consumption may be a potential source of infection for human.

\section{ACKNOWLEDGEMENTS}

The authors are grateful to the Ministry of Higher Education and Scientific Research of Benin, Through the Doctoral Support Program No. $125 \mathrm{MESRS} / \mathrm{CAB} / \mathrm{C} / \mathrm{SGM} / \mathrm{DRFM} / \mathrm{DRH} /$ REGIE of 24 February 2015.

\section{REFERENCES}

Akpovi, J., M. Kone, I. Takpara, R. Perrin, A. Massougbodji \& E. Alihonou, 1998. Grossesse et toxoplasmose à Cotonou. Le Bénin Médical Spécial Gynécologie et Obstérique, 8, 87-90.

Arko-Mensah, J., K. M. Bosompem, E. A. Canacoo, J. M. Wastling \& B. D. Akanmori, 2000. The seroprevalence of toxoplasmosis in pigs in Ghana. Acta Tropica, 76, 27-31.

Avode, D. G., C. Adjien, D. Houinato, M. Sounhin \& T. Adoukonou, 2005. Toxoplasmose cerebrale en milieu hospitalier à Cotonou (Benin). African Journal of Neurological Sciences, 24, 48.

Ayinmode, A. B. \& R. I. Olaosebikan, 2013. Antibodies to Toxoplasma gondii in backyard and wandering pigs in Ibadan, Nigeria: Implications for pork consumption. Bulletin of Animal Health and Production in Africa, 61, 493-497.

Bamba, S., L. Halos, Z. Tarnagda, A. Alanio, P. Macé, S. Moukoury \& S. Bretagne, 2016. Seroprevalence of Toxoplasma gondii and direct genotyping using minisequencing in free-range pigs in Burkina 
Faso. International Journal of Food Microbiology, 230, 10-15.

Dubey, J. P. \& J. L. Jones, 2008. Toxoplasma gondii infection in humans and animals in the United States. International Journal for Parasitology, 38, 1257-1278.

Dubey, J. P., 2009. Toxoplasmosis in pigs the last 20 years. Veterinary Parasitology, 164, 89-103.

Dubey, J. P., 2016. Toxoplasmosis of Animals and Humans, $2^{\text {nd }}$ edn, CRC Press, NewYork.

Gamble, H. R., J. P. Dubey \& D. N. Lambillotte, 2005. Comparison of a commercial ELISA with the modified agglutination test for detection of Toxoplasma infection in the domestic pig. Veterinary Parasitology, 128, 177-181.

Gebremedhin, E. Z., M. M. Kebeta, M. Asaye, H. Ashenafi, V. Di Marco \& M. Vitale, 2015. First report on seroepidemiology of Toxoplasma gondii infection in pigs in Central Ethiopia. BMC Veterinary Research, 11, 59.

Hill, D. E. \& J. P. Dubey, 2016. Toxoplasma gondii as a parasite in food: Analysis and control. Microbiology Spectrum, 4, doi:10.1128/microbiolspec.PFS-00112015.

Hove, T., P. Lind \& S. Mukaratirwa, 2005. Seroprevalence of Toxoplasma gondii infection in domestic pigs reared under different management systems in Zimbabwe. Onderstepoort Journal of Veterinary Research, 72, 231-237.

Kijlstra, A., B. Meerburg, J. Cornelissen, S. De Craeye, P. Vereijken \& E. Jongert, 2008. The role of rodents and shrews in the transmission of Toxoplasma gondii to pigs. Veterinary Parasitology, 156, 183 190.

Lindsay, D. S. \& L. M. Weiss, 2008. Opportunistic Infections: Toxoplasma, Sarcocystis, and Microsporidia. Springer Science \& Business Media.

Ogouyèmi-Hounto, A., F. AgbayahounChokki, Y. S. S de Tove, B. B. Bankole,
V. A. de Souza, M. Assogba \& A. Massougbodji, 2014. Évaluation d'un test de diagnostic rapide pour le diagnostic de la toxoplasmose chez les femmes enceintes à Cotonou (Bénin). Bulletin de la Société de Pathologie Exotique, 107, 85-89.

Peel, M. C., B. L. Finlayson \& T. A. Mcmahon, 2007. Updated world map of the Köppen-Geiger climate classification. $H y$ drology and Earth System Sciences Discussions, 4, 439-473.

Rakotoharinome, V. M., H. R. Andriamanivo, R. Blaga, C. Perret, S. A. Lacour \& A. Grasset-Chevillot, 2012. Toxoplasmosis and trichinellosis: An epidemiological survey of pig population in Madagascar. Revue d'Elevage et de Médecine Vétérinaire des Pays Tropicaux, 67, 113-114.

Tenter, A. M., A. R. Heckeroth, L. M. Weiss, 2000. Toxoplasma gondii: From animals to humans. International Journal for Parasitology, 30, 1217-1258.

Thrusfield, M. V., 2005. Criteria for success of questionnaire. Veterinary Epidemiology, 3, 189-213.

Tonouhewa, A. B. N., Y. Akpo, P. Sessou, C. Adoligbe, E. Yessinou, Y. G. Hounmanou, M. N. Assogba, I. Youssao \& S. Farougou, 2017. Toxoplasma gondii infection in meat animals from Africa: Systematic review and meta-analysis of sero-epidemiological studies. Veterinary World, 10, 194.

Villard, O., B. Cimon, C. L'Ollivier, H. Fricker-Hidalgo, N. Godineau, S. Houze \& E. Candolfi, 2016. Serological diagnosis of Toxoplasma gondii infection: Recommendations from the French National Reference Center for Toxoplasmosis. Diagnostic Microbiology and Infectious Disease, 84, 22.

Villena, I., B. Durand, D. Aubert, R. Blaga, R. Geers, M. Thomas \& A. Thébault, 2012. New strategy for the survey of Toxoplasma gondii in meat for human consumption. Veterinary Parasitology, 183, 203-208.

Wang, H., T. Wang, Q. Luo, X. Huo, L Wang \& T. Liu, 2012. Prevalence and genotypes of Toxoplasma gondii in pork from retail 
Toxoplasma gondii infections in pigs from south Benin and assessment of breeders' knowledge ....

meat stores in Eastern China. International Journal of Food Microbiology, 157, 393397.

Zannou, D. M., D. Kindé-Gazard, J. Vigan, G. Adè, J. J. Sèhonou, F. Atadokpèdé \& F. Houngbé, 2004. Profil clinique et immunologique des patients infectés par le VIH dépistés à Cotonou, Bénin. Médecine et Maladies Infectieuses, 34, 225-228.
Paper received 11.07.2018; accepted for publication 05.11.2018

\section{Correspondence:}

Aretas B. N. Tonouhewa

03BP 2253 Cotonou/Jericho, Benin, phone : 0022966823375 ,

e-mail: tonouhewaaretas@gmail.com 